\title{
Peripheral Blood Biomarkers Provide Prediction of Survival and Immune-Related Pneumonitis in Stage III/IV Non-Small Cell Lung Cancer Treated with Immune Checkpoint Inhibitors
}

\section{Haishan Wu}

Fujian Medical University Cancer Hospital, Fujian Cancer Hospital

\section{Yahua Wu}

Fujian Medical University Cancer Hospital, Fujian Cancer Hospital

\section{Mengyan Zhang}

Fujian Medical University Cancer Hospital, Fujian Cancer Hospital

Yilin Yu

Fujian Medical University Cancer Hospital, Fujian Cancer Hospital

Mingqiang Lin

Fujian Medical University Cancer Hospital, Fujian Cancer Hospital

\section{Zhiping Wang}

Fujian Medical University Cancer Hospital, Fujian Cancer Hospital

Tianxiu Liu

Fujian Medical University Cancer Hospital, Fujian Cancer Hospital

Qunhao Zheng

Fujian Medical University Cancer Hospital, Fujian Cancer Hospital

Jiayu Ma

Fujian Medical University Cancer Hospital, Fujian Cancer Hospital

Lingyun Liu

Fujian Medical University Cancer Hospital, Fujian Cancer Hospital

Jiancheng Li ( $\sim$ lijiancheng1212@126.com )

Fujian Medical University Cancer Hospital, Fujian Cancer Hospital

\section{Research Article}

Keywords: Non-small cell lung cancer, Immune checkpoint inhibitors, Peripheral blood biomarkers, Immune-related pneumonitis

Posted Date: June 10th, 2021 
DOl: https://doi.org/10.21203/rs.3.rs-583861/v1

License: (c) (1) This work is licensed under a Creative Commons Attribution 4.0 International License. Read Full License 


\section{Abstract}

Backgroud: Immune checkpoint inhibitors (ICls) have been considered as a standard therapy for nonsmall cell lung cancer (NSCLC). This study aimed to explore associations between peripheral blood biomarkers with survival and immune-related pneumonitis (IRP) in patients with stage III/IV NSCLC treated with ICls, and establish a novel risk stratification to predict outcomes.

Methods: This study enrolled 95 patients with stage III/IV NSCLC receiving ICls. Univariate and multivariate cox regression analyses were used to determine prognostic factors affecting overall survival (OS) and progression-free survival (PFS). Logistic regression analysis was applied to explore the correlation between peripheral blood markers and IRP.

Results: Multivariate Cox analysis revealed that pretreatment absolute lymphocyte count (ALC) (HR: $0.113,95 \% \mathrm{Cl}: 0.113-0.863, \mathrm{P}=0.025)$, and post-treatment systemic inflammation response index (SIRI) (HR: 4.199, 95\%Cl: 1.058-16.662, $\mathrm{P}=0.041$ ) were independent prognostic factors for OS. Similarly, pretreatment ALC (HR: 0.333, 95\%Cl: $0.129-0.857, \mathrm{P}=0.023)$ and post-treatment SIRI (HR: $3.160,95 \% \mathrm{Cl}$ : 1.046-9.551, $\mathrm{P}=0.041$ ) were also independent predictors of PFS. Based on these biomarkers, patients were classified into 3 categories: low-risk group: ALC >1.5 and SIRI $\leq 1.69(n=28)$; medium-risk group: ALC $>1.5$ or SIRI $\leq 1.69(n=35)$; high-risk group ALC $\leq 1.5$ and SIRI $>1.69(n=32)$. The 1-year OS rate were $69.2 \%, 63.6 \%, 27.1 \%$, respectively $(P=0.001)$ and 1 -year PFS rate were $51.8 \%, 44.8 \%, 21.1 \%$, respectively $(P=0.007)$. Furthermore, we found patients with post-treatment NLR $>3$ were associated with a significantly higher risk for occurring IRP than that NLR $\leq 3$ (HR: 2.917, 95\%Cl: 1.037-8.206, P=0.043), and the time to IRP onset in post-treatment NLR $>3$ group was significantly shorter than that in NLR $\leq 3$ $(P=0.018)$.

Conclusions: Pretreatment ALC and post-treatment SIRI appeared to be biomarkers of outcome in stage III/IV NSCLC patients treated with ICls. Furthermore, post-treatment NLR $>3$ was more likely to develop IRP.

\section{Backgroud}

Lung cancer is one of the most prevalent cancers globally and the incidence increases $26 \%$ annually. Non-small cell lung cancer (NSCLC), which is comprised of approximately $85 \%$ of all lung cancer patients. [1] And almost 75\% of the patients are diagnosed at stage III/IV NSCLC with the poor prognosis.[2]

Recently, immune-checkpoint inhibitors (ICls), anti-PD-1 (programmed cell death-1) and anti-PD-L1 (programmed cell death-Ligand 1) have demonstrated to improve long-term survival and considered as a novel standard treatment for advance non-small-cell lung cancer (NSCLC).[3-8] However, it was only approximately $20 \%$ patients who had positively response to ICls, and a minority of patients who could not benefit from ICls.[9] Furthermore, the use of ICls also commonly emerged immune-related adverse effects (irAEs),[10] one particular irAE is the development of immune-related pneumonitis (IRP), which is a rare but potentially fatal.[11-14] Therefore, it is important to select eligible patients who may benefit the ICls therapy and identify factors associated with the onset of IRP. 
Inflammatory response and immune surveillance have been deemed crucial signatures regarding cancer development and treatment outcomes.[15] A variety of evidence indicated that peripheral blood Inflammatory biomarkers such as neutrophil-to-lymphocyte ratio (NLR), platelet-to-lymphocyte ratio (PLR), lymphocyte-to-monocyte ratio (LMR), prognostic nutritional index (PNI), systemic inflammation index (SII), and systemic inflammation response index (SIRI) could facilitate the prediction of treatment outcomes for various solid carcinomas, including melanoma, prostate cancer, colorectal cancer, esophageal squamous cell carcinoma, and NSCLC.[16-20] These Inflammatory biomarkers are easy to obtain in clinical practice and directly reflect the condition of the immune system, and may potentially impact on the response to ICls.[20,21]

However, in currently, there were few reported studies on the predictive value of peripheral blood biomarkers for NSCLC treated with ICls. Therefore, we performed a retrospective analysis to investigate the usefulness of Inflammatory biomarkers including ANC, ALC, AMC, LMR, NLR, PLR, PNI, SII, and SIRI as predictors of outcomes and the development of IRP in stage III/IV NSCLC treated with ICls therapy.

\section{Patients And Methods}

\subsection{Patients}

We enrolled 95 patients with stage III/IV NSCLC who had treated with PD-1/PD-L1 inhibitors at Fujian Medical University Cancer Hospital between January 2017 and December 2020. The inclusion criteria were: (1) pathologically diagnosed with NSCLC; (2) clinical stage was III/IV; (3) KPS > 70; (4) received at least 1 cycle of $\mathrm{ICl}$ agents. The ICl agents used were nivolumab (12 patients), pembrolizumab (23 patients), atezolizumab (4 patients), toripalimab (2 patients), Sintilimab (38 patients), durvalumab (3 patients), tislelizumab (5 patients), camrelizumab (5 patients), nivolumab followed by pembrolizumab ( 1 patient), nivolumab followed by pembrolizumab and Sintilimab (1 patient), and nivolumab followed by toripalimab and durvalumab (1 patient). The exclusion criteria were: (1) patients with a histological type other than adenocarcinoma or squamous carcinoma (exclude 13 patients); (2) had a second primary malignancy; (3) hematological disease or autoimmune diseases; (4) insufficient clinical or laboratory data (exclude 14 patients). All patients were clinically staged using the American Joint Committee on Cancer (AJCC) 8th edition TNM staging system.[22] The study was approved by the ethics committee of Fujian Medical University Cancer Hospital, Fuzhou, China (YK2021-009-01).

\subsection{Data collection}

The clinical information included age, gender, karnofsky performance status (KPS), smoking history, tumor histology, clinical stage, PD-L1 expression, molecular alterations, treatment data, and peripheral blood biomarkers consisted of absolute neutrophil count (ANC), absolute lymphocyte count (ALC), absolute monocyte count (AMC), lymphocyte-to-monocyte ratio (LMR), neutrophil-to-lymphocyte ratio (NLR), platelet-to-lymphocyte (PLR), prognostic nutritional index (PNI), systemic inflammation index (SII), and systemic inflammation response index (SIRI). Peripheral blood biomarkers were collected from one week before the initial of ICls treatment and within 3-4 weeks after the initial of ICls treatment. The LMR, 
NLR, PLR, PNI, SII, and SIRI were calculated as follows: LMR = ALC / AMC, NLR = ANL / ALC, PLR = Platelet count $/$ ALC, PNI = 10×Serum Albumin level + $0.005 \times$ ALC, SII = ANC $\times$ Platelet count $/$ ALC, and SIRI $=$ ANC $\times$ AMC / ALC. All peripheral blood biomarkers cut-offs were obtained using the median value.

\subsection{Endpoints}

The primary endpoints included overall survival (OS) and progression-free survival (PFS). OS was defined as the time between initial ICls treatment and death for any reason or the last follow-up. PFS was measured from the first time of ICls treatment to the disease progression or death due to any cause. The secondary endpoint was the development of IRP. The time to onset of IRP was ranged from the start of ICls therapy to the development of IRP. The IRP was reviewed by the National Cancer Institute's Common Terminology Criteria for Adverse Events (CTCAE), version 5.0. All patients were followed up until death or the last follow-up. The median follow-up period was 10 months (range 2-38 months).

\subsection{Statistical analysis}

A Chi-squared test or Fisher exact test was used to compare categorical variables and a Mann-Whitney U test was used to compare continuous variables. Survival curves were estimated by the Kaplan-Meier method and compared by the log-rank test. Variables with a P-value $\leq 0.15$ in the univariate analysis was included in the multivariable analyses. Multivariate analysis was carried out to identify independent prognostic factors related to OS and PFS. Logistic regression analysis was applied to explore the correlation between peripheral blood markers and the onset of IRP. All analyses were considered as statistically significant with a two-sided P-value of <0.05, and were performed using SPSS 24.0 (IBM, Armonk, NY, USA).

\section{Results}

\subsection{The characteristics of patients}

A total of 95 NSCLC patients treated with ICls were included in our study. The characteristics of patients were given in Table 1. Of the 95 patients, 76 (80.0\%) were males, 34 (35.2\%) were $\geq 65$ years, 60 (57.0\%) had adenocarcinoma, and 75 (78.9\%) had a history of smoking. In addition, there were $37(38.9 \%)$ patients at stage III and 58 (61.9\%) at stage IV. The PD-L1 expression in 0, 1-49\%, and $\geq 50 \%$ were $5.3 \%$, $15.8 \%$, and $18.9 \%$, respectively. 4 patients had an EGFR mutation, 9 patients had a KRAS mutation, and none had ALK/ROS1 fusion. 31 (32.6\%) patients had received prior thoracic radiation therapy, 87 (91.6\%) had undergone chemotherapy, and $11(11.6 \%)$ had treated prior curable operation. ICls were received as first-line treatment in 42 patients (44.2\%), and as second-line or later-line treatment in 53 patients (55.8\%). 
Table 1

Characteristics of patients treated with ICls

\begin{tabular}{|c|c|}
\hline Variable & Patients treated with ICls $(n=95), n(\%)$ \\
\hline \multicolumn{2}{|l|}{ Age } \\
\hline$<65$ & $61(64.2)$ \\
\hline$\geq 65$ & $34(35.2)$ \\
\hline \multicolumn{2}{|l|}{ Gender } \\
\hline Male & $76(80.0)$ \\
\hline Female & 19(20.0) \\
\hline \multicolumn{2}{|l|}{ Smoking } \\
\hline Yes & 75(78.9) \\
\hline No & $20(21.1)$ \\
\hline \multicolumn{2}{|l|}{ KPS } \\
\hline 80 & $61(64.2)$ \\
\hline 90 & $34(35.8)$ \\
\hline \multicolumn{2}{|l|}{ Histology } \\
\hline Adenocarcinoma & $57(60.0)$ \\
\hline Squamous cell carcinoma & $38(40.0)$ \\
\hline \multicolumn{2}{|l|}{ Clinical stage } \\
\hline III & $37(38.9)$ \\
\hline IV & $58(61.9)$ \\
\hline \multicolumn{2}{|l|}{ Prior thoracic radiotherapy } \\
\hline Yes & $31(32.6)$ \\
\hline No & $64(67.4)$ \\
\hline \multicolumn{2}{|l|}{ Combine chemotherapy } \\
\hline Yes & $87(91.6)$ \\
\hline No & $8(8.4)$ \\
\hline \multicolumn{2}{|l|}{ Prior curable operation } \\
\hline Yes & $11(11.6)$ \\
\hline No & $84(88.4)$ \\
\hline
\end{tabular}




\begin{tabular}{|c|c|}
\hline Variable & Patients treated with ICls $(n=95), n(\%)$ \\
\hline \multicolumn{2}{|l|}{ Molecular alterations } \\
\hline EGFR or ALK/ROS1(+) & 13(13.7) \\
\hline$(-) /$ not done & $89(86.3)$ \\
\hline \multicolumn{2}{|l|}{ PD-L1 expression } \\
\hline 0 & $5(5.3)$ \\
\hline $1-49$ & $15(15.8)$ \\
\hline$\geq 50$ & 18(18.9) \\
\hline Not done & $57(52.6)$ \\
\hline \multicolumn{2}{|l|}{ Line of immunotherapy } \\
\hline First line & $42(44.2)$ \\
\hline Further lines & $53(55.8)$ \\
\hline \multicolumn{2}{|c|}{ Pretreatment peripheral blood biomarkers Median (IQR) } \\
\hline ANC & $4.9(3.5-6.3)$ \\
\hline ALC & $1.5(1.1-2.0)$ \\
\hline AMC & $0.51(0.39-0.69)$ \\
\hline LMR & $2.98(1.84-4.32)$ \\
\hline NLR & $3.15(2.21-5)$ \\
\hline PLR & $175(122.86-245)$ \\
\hline PNI & $45(40.1-51)$ \\
\hline SII & $846.61(514.8-1356)$ \\
\hline SIRI & $1.52(1.01-3.1)$ \\
\hline \multicolumn{2}{|c|}{ Post-treatment peripheral blood biomarkers Median (IQR) } \\
\hline ANC & $4.9(4-6.4)$ \\
\hline ALC & $1.5(1.1-1.9)$ \\
\hline AMC & $0.51(0.41-0.69)$ \\
\hline LMR & $3.12(2.2-4)$ \\
\hline NLR & $3.29(2.31-5.55)$ \\
\hline PLR & $175(121.36-247.14)$ \\
\hline
\end{tabular}




\begin{tabular}{|ll|}
\hline Variable & Patients treated with ICls $(\mathbf{n}=95), \mathbf{n}(\%)$ \\
\hline PNI & $46(40.6-49.5)$ \\
\hline SII & $846.40(527.06-1395.68)$ \\
SIRI & $1.69(1.09-3.08)$ \\
\hline
\end{tabular}

Abbreviations: ICls, immune checkpoint inhibitors; KPS, karnofsky performance status; PD-L1, programmed cell death-Ligand 1; IQR, interquartile range; ANC, absolute neutrophil count; ALC, absolute lymphocyte count; AMC, absolute monocyte count; LMR, lymphocyte-to-monocyte ratio; NLR, neutrophilto-lymphocyte ratio; PLR, platelet-to-lymphocyte; PNI, prognostic nutritional index; SIl, systemic inflammation index; SIRI, systemic inflammation response index.

\subsection{Univariate and multivariate cox analysis for PFS and OS}

Univariate and multivariate cox analysis for OS and PFS were detailed in Table 2 and Table 3 , respectively. As shown in Table 2, in Univariate cox analysis, the factors significantly correlated with OS were clinical stage $(P=0.023)$, prior thoracic radiotherapy $(P=0.014)$, Molecular alterations $(P=0.046)$, pretreatment NLR $(P=0.033)$, pretreatment PLR $(P=0.041)$, pretreatment $P N I(P=0.023)$, pretreatment SII $(P=0.013)$, post-treatment $L M R(P=0032)$, post-treatment $L M R(P=0.010)$, and post-treatment $S I R I(P=$ 0.018). In Multivariate Cox analysis, only prior thoracic radiotherapy (HR: $0.180,95 \% \mathrm{Cl}: 0.067-0.487, \mathrm{P}=$ 0.001), pretreatment ALC (HR: $0.113,95 \% \mathrm{Cl}: 0.113-0.863, \mathrm{P}=0.025)$, and post-treatment SIRI (HR: 4.199, 95\% Cl: $1.058-16.662, \mathrm{P}=0.041)$ were independent prognostic factors for OS. Similarly, the multivariate cox analysis indicated that prior thoracic radiotherapy (HR: $0.239,95 \% \mathrm{Cl}: 0.100-0.570, \mathrm{P}=0.001$ ), pretreatment ALC (HR: $0.333,95 \% \mathrm{Cl}: 0.129-0.857, \mathrm{P}=0.023)$, pretreatment PNI (HR: $2.750,95 \% \mathrm{Cl}: 1.033-$ 7.322, $\mathrm{P}=0.043$ ), post-treatment PLR (HR: $2.213,95 \% \mathrm{Cl}: 1.039-4.715, \mathrm{P}=0.040)$, and post-treatment SIRI (HR: $3.160,95 \% \mathrm{Cl}: 1.046-9.551, \mathrm{P}=0.041$ ) were independent predictors of PFS (Table 3). 
Table 2

Univariate and multivariate cox analyses of factors associated with overall survival in NSCLC patients treated with ICls.

\begin{tabular}{|c|c|c|}
\hline \multirow[t]{2}{*}{ Variable } & Univariate analysis & Multivariate analysis \\
\hline & HR (95\% Cl), P value & HR (95\% Cl), P value \\
\hline Age $(<65$ vs. $\geq 65)$ & $\begin{array}{l}1.269(0.716-2.247) \\
0.414\end{array}$ & \\
\hline Gender(Female vs. Male) & $\begin{array}{l}0.988(0.472-2.067) \\
0.974\end{array}$ & \\
\hline Smoking(No vs. Yes) & $\begin{array}{l}1.129(0.541-2.354) \\
0.746\end{array}$ & \\
\hline KPS(80 vs. 90) & $\begin{array}{l}1.218(0.691-2.149) \\
0.495\end{array}$ & \\
\hline Histology(Squamous vs. Adenocarcinoma) & $\begin{array}{l}0.876(0.495-1.550) \\
0.649\end{array}$ & \\
\hline Clinical stage(III vs. IV) & $\begin{array}{l}2.129(1.109-4.087) \\
0.023\end{array}$ & $\begin{array}{l}1.551(0.630-3.820) \\
0.340\end{array}$ \\
\hline Prior thoracic radiotherapy(No vs. Yes) & $\begin{array}{l}0.426(0.216-0.841) \\
0.014\end{array}$ & $\begin{array}{l}0.180(0.067-0.487), \\
0.001\end{array}$ \\
\hline Combine chemotherapy(No vs. Yes) & $\begin{array}{l}1.500(0.462-4.867) \\
0.500\end{array}$ & \\
\hline Prior curable operation(No vs. Yes) & $\begin{array}{l}0.412(0.128-1.328) \\
0.138\end{array}$ & $\begin{array}{l}0.501(0.114-2.206), \\
0.361\end{array}$ \\
\hline Molecular alterations(No/not done vs. Yes) & $\begin{array}{l}2.115(1.014-4.410) \\
0.046\end{array}$ & $\begin{array}{l}2.321(0.903-5.965), \\
0.080\end{array}$ \\
\hline PD-L1 expression & - & \\
\hline 0 vs. $1-49$ & $\begin{array}{l}0.894(0.244-3.276) \\
0.866\end{array}$ & \\
\hline 0 vs. $\geq 50$ & $\begin{array}{l}1.261(0.343-4.634) \\
0.727\end{array}$ & \\
\hline $\begin{array}{l}\text { Line of immunotherapy(First line vs. Further } \\
\text { lines) }\end{array}$ & $\begin{array}{l}1.002(0.570-1.761) \\
0.994\end{array}$ & \\
\hline \multicolumn{3}{|l|}{ Pretreatment peripheral blood biomarkers } \\
\hline $\mathrm{ANC}(\leq 4.9$ vs. $>4.9)$ & $\begin{array}{l}1.593(0.893-2.841) \\
0.115\end{array}$ & $\begin{array}{l}1.547(0.622-3.848) \\
0.348\end{array}$ \\
\hline $\operatorname{ALC}(\leq 1.5$ vs. $>1.5)$ & $\begin{array}{l}0.574(0.320-1.027) \\
0.062\end{array}$ & $\begin{array}{l}0.312(0.113-0.863) \\
0.025\end{array}$ \\
\hline
\end{tabular}




\begin{tabular}{|c|c|c|}
\hline \multirow[t]{2}{*}{ Variable } & Univariate analysis & Multivariate analysis \\
\hline & HR (95\% Cl), $\mathrm{P}$ value & HR ( $95 \% \mathrm{Cl}), \mathrm{P}$ value \\
\hline $\mathrm{AMC}(\leq 0.51$ vs. $>0.51)$ & $\begin{array}{l}1.218(0.684-2.168) \\
0.503\end{array}$ & \\
\hline $\operatorname{LMR}(\leq 2.98$ vs. $>2.98)$ & $\begin{array}{l}0.629(0.357-1.110) \\
0.110\end{array}$ & $\begin{array}{l}1.135(0.380-3.391) \\
0.821\end{array}$ \\
\hline $\operatorname{NLR}(\leq 3.15$ vs. $>3.15)$ & $\begin{array}{l}1.859(1.051-3.290) \\
0.033\end{array}$ & $\begin{array}{l}1.244(0.361-4.292) \\
0.729\end{array}$ \\
\hline PLR( $\leq 175$ vs. >175) & $\begin{array}{l}1.810(1.024-3.198) \\
0.041\end{array}$ & $\begin{array}{l}0.681(0.264-1.754), \\
0.426\end{array}$ \\
\hline $\mathrm{PNI}(\leq 45$ vs. $>45)$ & $\begin{array}{l}0.512(0.287-0.910) \\
0.023\end{array}$ & $\begin{array}{l}2.684(0.893-8.068) \\
0.079\end{array}$ \\
\hline $\operatorname{SII}(\leq 846.61$ vs. $>846.61)$ & $\begin{array}{l}2.064(1.162-3.665) \\
0.013\end{array}$ & $\begin{array}{l}3.562(0.888-14.286) \text {, } \\
0.073\end{array}$ \\
\hline $\operatorname{SIRI}(\leq 1.52$ vs. $>1.52)$ & $\begin{array}{l}1.533(0.869-2.704) \\
0.140\end{array}$ & $\begin{array}{l}0.262(0.096-1.360) \\
0.132\end{array}$ \\
\hline \multicolumn{3}{|c|}{ Post-treatment peripheral blood biomarkers } \\
\hline $\operatorname{ANC}(\leq 4.9$ vs. $>4.9)$ & $\begin{array}{l}1.733(0.978-3.073) \\
0.060\end{array}$ & $\begin{array}{l}1.737(0.675-4.467) \\
0.252\end{array}$ \\
\hline $\operatorname{ALC}(\leq 1.5$ vs. $>1.5)$ & $\begin{array}{l}0.721(0.408-1.275) \\
0.261\end{array}$ & \\
\hline $\operatorname{AMC}(\leq 0.51$ vs. $>0.51)$ & $\begin{array}{l}1.197(0.682-2.099) \\
0.531\end{array}$ & \\
\hline $\operatorname{LMR}(\leq 3.12$ vs. $>3.12)$ & $\begin{array}{l}0.534(0.301-0.948) \\
0.032\end{array}$ & $\begin{array}{l}1.109(0.348-3.532) \\
0.861\end{array}$ \\
\hline $\mathrm{NLR}(\leq 2.31$ vs. $>2.31)$ & $\begin{array}{l}1.373(0.773-2.437) \\
0.279\end{array}$ & \\
\hline $\operatorname{PLR}(\leq 175$ vs. $>175)$ & $\begin{array}{l}1.752(0.991-3.095) \\
0.054\end{array}$ & $\begin{array}{l}2.477(0.969-6.181) \\
0.058\end{array}$ \\
\hline $\mathrm{PNI}(\leq 46$ vs. $>46)$ & $\begin{array}{l}0.581(0.326-1.036) \\
0.066\end{array}$ & $\begin{array}{l}0.823(0.333-2.033) \\
0.673\end{array}$ \\
\hline SII $(\leq 846.4$ vs. $>846.4)$ & $\begin{array}{l}2.149(1.205-3.832) \\
0.010\end{array}$ & $\begin{array}{l}0.347(0.103-1.166) \\
0.087\end{array}$ \\
\hline $\operatorname{SIRI}(\leq 1.69$ vs. $>1.69)$ & $\begin{array}{l}1.992(1.125-3.527), \\
0.018\end{array}$ & $\begin{array}{l}4.199 \text { (1.058-16.662), } \\
0.041\end{array}$ \\
\hline
\end{tabular}

Abbreviations: NSCLC, non-small lung cancer; HR, hazard ratio; $\mathrm{Cl}$, confidence interval; KPS, karnofsky performance status; PD-L1, programmed cell death-Ligand 1; ANC, absolute neutrophil count; ALC, 
absolute lymphocyte count; AMC, absolute monocyte count; LMR, lymphocyte-to-monocyte ratio; NLR, neutrophil-to-lymphocyte ratio; PLR, platelet-to-lymphocyte; PNI, prognostic nutritional index; SII, systemic inflammation index; SIRI, systemic inflammation response index. 
Table 3

Univariate and multivariate cox analyses of factors associated with progress-free survival in NSCLC patients treated with ICls.

\begin{tabular}{|c|c|c|}
\hline \multirow[t]{2}{*}{ Variable } & Univariate analysis & Multivariate analysis \\
\hline & HR (95\% Cl), P value & HR (95\% Cl), P value \\
\hline Age $(<65$ vs. $\geq 65)$ & $\begin{array}{l}1.266(0.744-2.155) \\
0.385\end{array}$ & \\
\hline Gender(Female vs. Male) & $\begin{array}{l}1.450(0.683-3.076) \\
0.333\end{array}$ & \\
\hline Smoking(No vs. Yes) & $\begin{array}{l}1.631(0.770-3.454) \\
0.201\end{array}$ & \\
\hline KPS(80 vs. 90) & $\begin{array}{l}1.218(0.691-2.149) \\
0.495\end{array}$ & \\
\hline Histology(Squamous vs. Adenocarcinoma) & $\begin{array}{l}0.902(0.553-1.526) \\
0.702\end{array}$ & \\
\hline Clinical stage(III vs. IV) & $\begin{array}{l}1.689(0.958-2.975) \\
0.070\end{array}$ & $\begin{array}{l}1.023(0.471-2.221) \\
0.954\end{array}$ \\
\hline Prior thoracic radiotherapy(No vs. Yes) & $\begin{array}{l}0.507(0.277-0.928) \\
0.028\end{array}$ & $\begin{array}{l}0.239(0.100-0.570) \\
0.001\end{array}$ \\
\hline Combine chemotherapy(No vs. Yes) & $\begin{array}{l}2.009(0.624-6.467), \\
0.242\end{array}$ & \\
\hline Prior curable operation(No vs. Yes) & $\begin{array}{l}0.426(0.154-1.181), \\
0.101\end{array}$ & $\begin{array}{l}0.327(0.095-1.126), \\
0.076\end{array}$ \\
\hline Molecular alterations(No/not done vs. Yes) & $\begin{array}{l}1.958(0.983-3.901) \\
0.056\end{array}$ & $\begin{array}{l}2.145(0.956-4.814), \\
0.064\end{array}$ \\
\hline \multicolumn{3}{|l|}{ PD-L1 expression } \\
\hline 0 vs. $1-49$ & $\begin{array}{l}0.522(0.172-1.581) \\
0.250\end{array}$ & \\
\hline 0 vs. $\geq 50$ & $\begin{array}{l}0.804(0.275-2.354) \\
0.691\end{array}$ & \\
\hline $\begin{array}{l}\text { Line of immunotherapy(First line vs. Further } \\
\text { lines) }\end{array}$ & $\begin{array}{l}0.936(0.555-1.578) \\
0.805\end{array}$ & \\
\hline \multicolumn{3}{|l|}{ Pretreatment peripheral blood biomarkers } \\
\hline $\mathrm{ANC}(\leq 4.9$ vs. $>4.9)$ & $\begin{array}{l}1.552(0.916-2.630) \\
0.102\end{array}$ & $\begin{array}{l}1.287(0.639-2.589) \\
0.480\end{array}$ \\
\hline $\operatorname{ALC}(\leq 1.5$ vs. $>1.5)$ & $\begin{array}{l}0.605(0.605-1.030) \\
0.064\end{array}$ & $\begin{array}{l}0.333(0.129-0.857) \\
0.023\end{array}$ \\
\hline
\end{tabular}




\begin{tabular}{|c|c|c|}
\hline \multirow[t]{2}{*}{ Variable } & Univariate analysis & Multivariate analysis \\
\hline & HR (95\% Cl), $\mathrm{P}$ value & HR (95\% Cl), P value \\
\hline $\mathrm{AMC}(\leq 0.51$ vs. $>0.51)$ & $\begin{array}{l}1.319(0.776-2.240) \\
0.306\end{array}$ & \\
\hline $\operatorname{LMR}(\leq 2.98$ vs. $>2.98)$ & $\begin{array}{l}0.665(0.395-1.121) \\
0.125\end{array}$ & $\begin{array}{l}1.330(0.605-2.925), \\
0.478\end{array}$ \\
\hline NLR( 3.15 vs. >3.15) & $\begin{array}{l}1.437(0.854-2.418), \\
0.172\end{array}$ & \\
\hline $\operatorname{PLR}(\leq 175$ vs. $>175)$ & $\begin{array}{l}1.579(0.938-2.658) \\
0.086\end{array}$ & $\begin{array}{l}0.810(0.344-1.911), \\
0.631\end{array}$ \\
\hline $\mathrm{PNI}(\leq 45$ vs. $>45)$ & $\begin{array}{l}0.640(0.380-1.077), \\
0.093\end{array}$ & $\begin{array}{l}2.750(1.033-7.322), \\
0.043\end{array}$ \\
\hline SII $(\leq 846.61$ vs. $>846.61)$ & $\begin{array}{l}1.727(1.024-2.910), \\
0.040\end{array}$ & $\begin{array}{l}1.802(0.669-4.855), \\
0.244\end{array}$ \\
\hline $\operatorname{SIRI}(\leq 1.52$ vs. $>1.52)$ & $\begin{array}{l}1.392(0.829-2.336), \\
0.211\end{array}$ & \\
\hline \multicolumn{3}{|c|}{ Post-treatment peripheral blood biomarkers } \\
\hline $\mathrm{ANC}(\leq 4.9$ vs. $>4.9)$ & $\begin{array}{l}1.209(0.715-2.047), \\
0.479\end{array}$ & \\
\hline $\mathrm{ALC}(\leq 1.5$ vs. $>1.5)$ & $\begin{array}{l}0.703(0.416-1.188), \\
0.188\end{array}$ & \\
\hline $\operatorname{AMC}(\leq 0.51$ vs. $>0.51)$ & $\begin{array}{l}1.159(0.692-1.941), \\
0.576\end{array}$ & \\
\hline $\operatorname{LMR}(\leq 3.12$ vs. $>3.12)$ & $\begin{array}{l}0.613(0.364-1.035) \\
0.067\end{array}$ & $\begin{array}{l}1.080(0.429-2.719), \\
0.870\end{array}$ \\
\hline $\mathrm{NLR}(\leq 2.31$ vs. >2.31) & $\begin{array}{l}1.188(0.702-2.010), \\
0.521\end{array}$ & \\
\hline $\operatorname{PLR}(\leq 175$ vs. $>175)$ & $\begin{array}{l}1.518(0.902-2.554) \\
0.116\end{array}$ & $\begin{array}{l}2.213(1.039-4.715), \\
0.040\end{array}$ \\
\hline $\mathrm{PNI}(\leq 46$ vs. $>46)$ & $\begin{array}{l}0.689(0.412-1.185) \\
0.183\end{array}$ & \\
\hline SII $(\leq 846.4$ vs. $>846.4)$ & $\begin{array}{l}1.689(1.001-2.849), \\
0.050\end{array}$ & $\begin{array}{l}0.477(0.204-1.119), \\
0.089\end{array}$ \\
\hline $\operatorname{SIRI}(\leq 1.69$ vs. $>1.69)$ & $\begin{array}{l}1.719(1.021-2.895), \\
0.042\end{array}$ & $\begin{array}{l}3.160(1.046-9.551), \\
0.041\end{array}$ \\
\hline
\end{tabular}

\subsection{Immune prognostic factors for risk stratification}


Pretreatment ALC and post-treatment SIRI were considered as significant favorable prognostic factors for both OS and PFS. The Kaplan-Meier curves for OS and PFS were presented in Fig. 1. The median OS and PFS among patients who had post-treatment SIRI $>1.69$ were significantly shorter than that posttreatment $\mathrm{SIRI} \leq 1.69$ (OS: 5 months vs 11 months, $\mathrm{P}=0.013$; PFS: 3 months vs 8 months, $P=0.031$ ). Furthermore, the median OS and PFS among patients who had pretreatment ALC $>1.5$ was longer than that pretreatment $A L C \leq 1.5$ (OS: 9 months vs 7 months, $P=0.052$; PFS: 7 months vs 5 months, $P=$ 0.050). Based on these risk factors, we then classified patients into 3 categories: low-risk group: ALC > 1.5 and $\operatorname{SIRI} \leq 1.69(\mathrm{n}=28)$; medium-risk group: $\mathrm{ALC}>1.5$ or $\mathrm{SIRI} \leq 1.69(\mathrm{n}=35)$; high-risk group ALC $\leq 1.5$ and $\mathrm{SIRI}>1.69(\mathrm{n}=32)$. Kaplan-Meier analysis showed the 1-year OS rates of $69.2 \%, 63.6 \%, 27.1 \%$ in lowrisk, medium-risk, and high-risk group respectively $(P=0.001)$ (Fig. $2 A)$. In addition, the PFS of high-risk group were also significantly shorter than that of patients in low-risk or medium-risk group $(P=0.007)$ (Fig. 2B).

\subsection{The associations between IRP with peripheral blood biomarkers and outcomes}

In our study, 24 patients developed IRP attributed to PD-1/PD-L1 inhibitors. Among these patients, 12.5\% $(3 / 24)$ had grade $1,66.7 \%(16 / 24)$ had grade $2,12.5 \%(3 / 24)$ had grade 3 , and $8.3 \%(2 / 24)$ had grade 4 . As showed in Table 4, patients with IRP had significantly higher post-treatment NLR than those without IRP (the medium 4.23 versus $3.07, \mathrm{P}=0.045$ ). Logistic regression analysis (Table 5) revealed that posttreatment NLR was a risk factor for the occurrence of IRP. Patients with post-treatment NLR $>3$ were associated with a significantly higher risk for IRP than that NLR $\leq 3$ (HR: $2.917,95 \% \mathrm{Cl}: 1.037-8.206, \mathrm{P}=$ 0.043). 
Table 4

Characteristics among patients who with or without IRP.

\begin{tabular}{|c|c|c|c|}
\hline Variable & IRP $(n=24)$ & No IRP $(n=71)$ & P Value \\
\hline Age & & & 0.487 \\
\hline$<65$ & $14(58.3)$ & $47(66.2)$ & \\
\hline$\geq 65$ & $10(41.7)$ & 24(33.8) & \\
\hline Gender & & & 0.773 \\
\hline Male & $20(83.3)$ & $56(78.9)$ & \\
\hline Female & $4(16.7)$ & 15(21.1) & \\
\hline Smoking & & & 0.385 \\
\hline Yes & 21(87.5) & $54(76.1)$ & \\
\hline No & $3(12.5)$ & 17(23.9) & \\
\hline KPS & & & 0.772 \\
\hline 80 & $16(66.7)$ & $45(63.4)$ & \\
\hline 90 & 8(33.3) & $26(36.6)$ & \\
\hline Histology & & & 0.247 \\
\hline Adenocarcinoma & $12(50.0)$ & $45(63.4)$ & \\
\hline Squamous cell carcinoma & $12(50.0)$ & $26(36.6)$ & \\
\hline Clinical stage & & & 0.424 \\
\hline III & $11(45.8)$ & $26(36.6)$ & \\
\hline IV & $13(54.2)$ & $45(63.4)$ & \\
\hline Prior thoracic radiotherapy & & & 0.275 \\
\hline Yes & $10(41.7)$ & 21(29.6) & \\
\hline No & $14(58.3)$ & $50(70.4)$ & \\
\hline Combine chemotherapy & & & 1.000 \\
\hline Yes & $22(91.7)$ & $65(91.5)$ & \\
\hline No & $2(8.3)$ & $6(8.5)$ & \\
\hline Prior curable operation & & & 1.000 \\
\hline Yes & $3(12.5)$ & $8(11.3)$ & \\
\hline No & $21(87.5)$ & 63(88.7) & \\
\hline
\end{tabular}




\begin{tabular}{|c|c|c|c|}
\hline Variable & IRP $(n=24)$ & No IRP $(n=71)$ & P Value \\
\hline Molecular alterations & & & 1.000 \\
\hline EGFR or ALK/ROS1(+) & $3(12.5)$ & $10(14.1)$ & \\
\hline$(-) /$ not done & $21(87.5)$ & $61(85.9)$ & \\
\hline PD-L1 expression & & & 0.134 \\
\hline 0 & 2(8.3) & $3(4.2)$ & \\
\hline $1-49$ & $4(16.7)$ & $11(15.5)$ & \\
\hline$\geq 50$ & $8(33.3)$ & $10(14.1)$ & \\
\hline Not done & 14(58.3) & 24(33.8) & \\
\hline Line of immunotherapy & & & 0.256 \\
\hline First line & $13(54.2)$ & $29(40.8)$ & \\
\hline Further lines & $11(45.8)$ & $42(59.2)$ & \\
\hline \multicolumn{4}{|c|}{ Pretreatment peripheral blood biomarkers Median (IQR) } \\
\hline ANC & $4.35(3.38-6.78)$ & $4.9(3.5-6.2)$ & 0.448 \\
\hline ALC & $1.40(1.20-1.80)$ & $1.5(1.1-2)$ & 0.938 \\
\hline AMC & $0.51(0.41-0.76)$ & $0.51(0.37-0.68)$ & 0.700 \\
\hline LMR & $3.00(2.17-3.92)$ & $2.91(1.69-4.39)$ & 0.956 \\
\hline NLR & $3.11(2.23-4.64)$ & $3.19(2.2-5.74)$ & 0.771 \\
\hline PLR & $163.61(123.04-240.19)$ & $178.33(122.35-246.67)$ & 0.675 \\
\hline PNI & $46.25(42.35-50.1)$ & $44.6(39.9-51.7)$ & 0.647 \\
\hline SII & $897.58(430.73-1254)$ & $775.38(526.5-1584.6)$ & 0.613 \\
\hline SIRI & $1.60(1.12-2.31)$ & $1.51(1.00-3.12)$ & 0.794 \\
\hline \multicolumn{4}{|c|}{ Post-treatment peripheral blood biomarkers Median (IQR) } \\
\hline ANC & $5.7(4.45-7.80)$ & $4.7(3.7-6.1)$ & 0.059 \\
\hline ALC & $1.40(0.73-2.35)$ & $1.5(1.2-1.8)$ & 0.487 \\
\hline AMC & $0.55(0.28-0.74)$ & $0.51(0.42-0.68)$ & 0.861 \\
\hline LMR & $3.12(2.07-3.91)$ & $3.12(2.2-4.2)$ & 0.837 \\
\hline NLR & $4.23(2.97-7.63)$ & $3.07(2.23-5.13)$ & 0.045 \\
\hline PLR & $170.72(103.33-314.33)$ & $180(12.35-245)$ & 0.820 \\
\hline
\end{tabular}




\begin{tabular}{|llll|}
\hline Variable & IRP $(\boldsymbol{n}=\mathbf{2 4})$ & No IRP $(\boldsymbol{n}=\mathbf{7 1})$ & P Value \\
\hline PNI & $46(39.10-50.35)$ & $46(41.2-49.5)$ & 0.706 \\
SII & $937.57(656.70-1552.11)$ & $790.86(522.2-1378.91)$ & 0.262 \\
SIRI & $2.4(1.22-3.31)$ & $1.56(1.04-2.71)$ & 0.134 \\
\hline
\end{tabular}

Abbreviations: IRP, immune-related pneumonitis; KPS, karnofsky performance status; PD-L1, programmed cell death-Ligand 1; IQR, interquartile range; ANC, absolute neutrophil count; ALC, absolute lymphocyte count; AMC, absolute monocyte count; LMR, lymphocyte-to-monocyte ratio; NLR, neutrophil-tolymphocyte ratio; PLR, platelet-to-lymphocyte; PNI, prognostic nutritional index; SII, systemic inflammation index; SIRI, systemic inflammation response index.

We also found that among patients with post-treatment NLR $>3,3(16.7 \%)$ had grade 1 ICl-pneumonitis, $10(55.6 \%)$ had grade $2,3(16.7 \%)$ had grade 3 , and $2(11.1 \%)$ had grade 4 . For post-treatment NLR $\leq 3$, all patient developed grade $2 \mathrm{ICl}$-pneumonitis. But it was no significant difference in the distribution of grade between post-treatment NLR $>3$ group and post-treatment NLR $\leq 3$ group $(P=0.280)$ (Fig. $3 A)$. In addition, we observed that most of patients (18/24) developed IRP within the first 3 months after the start of ICls treatment, and the time to IRP onset in post-treatment NLR $>3$ group was shorter than in posttreatment NLR $\leq 3$ group (Fig. 3B). The median onset time of IRP was 2 months (range 1-18 months), and the median IRP onset time in post-treatment NLR $>3$ group was 1.5 months (range 1-16 months), and 6.5 months (range 1-18 months) in post-treatment NLR $\leq 3$ group.

Table 5

Univariate logistic regression analysis was performed to identify the risk of IRP in different cut-off values of post-treatment NLR.

\begin{tabular}{|lll|}
\hline Variable & HR $(95 \%$ Cl $)$ & p-value \\
\hline Post-treatment NLR $(\leq 2$ vs. $>2)$ & $1.875(0.492-7.141)$ & 0.357 \\
\hline Post-treatment NLR $(\leq 3$ vs. $>3)$ & $2.917(1.037-8.206)$ & 0.043 \\
\hline Post-treatment NLR $(\leq 4$ vs. $>4)$ & $2.314(0.903-5.934)$ & 0.081 \\
\hline Post-treatment NLR $(\leq 5$ vs. $>5)$ & $1.472(0.540-4.014)$ & 0.450 \\
\hline Post-treatment NLR( $\leq 6$ vs. $>6)$ & $2.727(0.940-7.909)$ & 0.065 \\
\hline
\end{tabular}

Median OS and PFS were similar in patients who developed IRP compared with those who did not, however, patients with grade $\geq 3$ IRP were significantly associated with shorter OS (8 months versus 4 months, $P=0.04)$ and PFS (6.5 months versus 3 months, $P=0.054)$ (Fig. 4).

\section{Discussion}


The role of immunotherapy in the treatment of NSCLC has been increasingly emphasized, and its application in clinical practice largely changes the treatment and prognosis of NSCLC patients. $[4-6,8]$ In this study, a total of 95 stage III/IV NSCLC patients treated with ICls therapy was retrospectively analyzed. We found that both pretreatment ALC and post-treatment SIRI were useful predictors of outcomes in NSCLC patients receiving ICls. Then we established a novel risk stratification based on these prognostic factors. To our knowledge, this is the first study to combine pretreatment ALC with post-treatment SIRI for predicting outcomes in patients treated with ICls. Furthermore, our results showed that post-treatment NLR was a risk factor for the occurrence of IRP.

Currently, a growing body of studies illustrated that the clinical value of peripheral blood biomarkers which could be helpful for predicting treatment outcomes in different solid tumors, including NSCLC.[1620] Lymphocyte is one of peripheral blood biomarkers which is a vital indicator of the immune system, reflecting the immune system activation, and play a fundamentally important role in tumor proliferation and migration.[23-26] Previous studies have reported that the lymphopenia correlated with worse outcomes in cancer patients.[27-29] Ryoko et.al. found that pretreatment ALC less than 1.5 associated with adverse survival.[28] Another analysis of patients with advanced breast cancer, sarcoma, and lymphoma, suggested that lymphopenia was an independent predictor of survival.[29] In our study, we found that pretreatment ALC less than 1.5 predicted adverse OS and PFS for patients treated with ICls. The mechanism of cancer immunotherapy is to promote the activity of cytotoxic T lymphocytes (CTLs) and assist in the activation of tumor-specific CTLs in lymphoid organs, thus establishing an efficient and durable anti-tumor immune microenvironment for anti-tumor therapy.[30] Therefore, it is not difficult to understand that a higher pretreatment ALC is beneficial to improve the survival of patients receiving ICls.

Another peripheral blood biomarker is the systemic inflammation response index (SIRI), which combined ANC, ALC, and AMC, has been proved to be an effective prognostic biomarkers in different cancers such as esophageal squamous cell carcinoma, non-small cell lung cancer, pancreatic cancer, gastric adenocarcinoma, clear cell renal cell carcinoma.[20,31-34] Geng et al. suggested that SIRI was an independent prognostic indicator for ESCC patients after the radical surgery.[33] and Hu et al. proposed pretreatment SIRI was an independent predictor of outcomes in stage III NSCLC patients who undergoing chemoradiotherapy.[20] But few studies accessed the effect of SIRI for patients receiving immunotherapy. As far as we know, the present study was the first to demonstrate predictive roles of SIRI for prognosis of III/IV stage NSCLC patients treated with ICls and revealed that high post-treatment SIRI was significantly associated with poor OS and PFS. Then, we established a novel risk stratification based on pretreatment ALC and post-treatment SIRI. Patients were classified into 3 categories: low-risk group: ALC $>1.5$ and SIRI $\leq 1.69$; medium-risk group: $\mathrm{ALC}>1.5$ or $\mathrm{SIRI} \leq 1.69$; high-risk group: $\mathrm{ALC} \leq 1.5$ and $\mathrm{SIRI}>1.69$. Survival analysis showed 1-year OS rates of $69.2 \%, 63.6 \%, 27.1 \%$ in low-risk, medium-risk, and high-risk group respectively $(P=0.001)$. In addition, PFS of high-risk group were also significantly shorter than that patients in low-risk or medium-risk group $(P=0.007)$. Given that ALC and SIRI are easily obtainable, a simple immune risk stratification based on these factors may easily predict survival in III/IV NSCLC treated with ICls in clinical practice. 
Although immunotherapy has brought hope to patients with advanced tumors in recent years, the immune-related adverse effects (irAEs) it brings have also drawn attention.[10] Immune-related pneumonitis (IRP) is a rare but potential fatal irAE that is related to poor outcomes.[11-14] It reported that the incidence rate of all-grade and grade 3 or more IRP were almost $5.4-19.0 \%$ and $2.6-12.2 \%$ respectively in the clinical setting.[35-37] Our study presented a real-world observation concerning the onset of IRP by ICls therapy in clinical practice. 24 (25.2\%) patients developed IRP attributed to PD-1/PDL1 inhibitors. Among these patients, 12.5\% (3/24) had grade 1 IRP, $66.7 \%(16 / 24)$ had grade 2 IRP, $12.5 \%$ $(3 / 24)$ had grade 3 IRP, and 8.3\% (2/24) had grade 4 IRP. Median OS and PFS were similar in patients who developed IRP compared with those who did not, however, patients with grade $\geq 3$ IRP were associated with shorter OS ( 8 months versus 4 months, $p=0.04$ ) and PFS ( 6.5 months versus 3 months, $p=0.054)$.

Given severity and worse outcomes of IRP, many scholars have tried to identify reliable predictors for the occurrence of IRP. They suggested that IRP may be associated with tumor histology types, smoking history, presence of preexisting interstitial lung disease.[38, 39] However, no reliable predictive biomarkers were currently used to predict the risk of IRP onset. Therefore, our study also explored an association between IRP and the peripheral blood biomarkers. Intriguingly, we observed that higher post-treatment NLR showed an increased the IRP onset. Patients with post-treatment NLR $>3$ were corrected with a significantly higher risk for developing IRP than that post-treatment NLR $\leq 3$ (HR: 2.917, 95\% Cl: 1.0378.206, $P=0.043)$. There were few studies indicated that the NLR reflected the systemic immune status and may be regarded as a predictor of IRP in patients with ICls therapy.[40, 41] A study by Ryosuke et.al. confirmed that NLR could well predict the onset and severity of IRP. They also showed that a considerable elevated NLR during the development of IRP.[40] Furthermore, Fujisawa et al. Also found similar results of elevated neutrophils and decreased lymphocytes in grade 3 and 4 IRP.[41] The precise mechanism of higher NLR associated with the development of IRP is still unclear, and further in-depth studies are needed to validate our results and elucidate the mechanisms it involved.

In addition, we also explored the correction between NLR and the time to IRP onset, and found that most of patients developed IRP within the first 3 months after the start of ICls treatment, and the onset time in post-treatment NLR $>3$ group was shorter than in post-treatment NLR $\leq 3$ group. The median onset time in post-treatment NLR $>3$ group was 1.5 months (range 1-16 months), and 6.5 months (range 1-18 months) in post-treatment NLR $\leq 3$ group. Therefore, for those patients with post-treatment NLR $>3$, we should be alert to the development of IRP in the first 3 months after ICls therapy and adopt a timely treatment strategy.

The present study has several limitations. Firstly, this is a retrospective study, so there may be selective bias in our study. Secondly, there are small numbers of eligible patients, and all patients come from a single institution. Accordingly, larger prospective studies are needed to confirm our results. Third, our current study only explored parameters that are commonly used and easily accessible in clinical practice, but other relevant variables involving genomics and radiomics may provide more valuable information to improve the predictive accuracy of IRP as well as the prognosis of patients treated with ICls. 


\section{Conclusion}

Our data indicated that pretreatment ALC and post-treatment SIRI appeared to be biomarkers of outcome in stage III/IV NSCLC patients treated with ICls. A novel risk stratification was established basing on these prognostic factors. Patients were classified into 3 categories: low-risk group: ALC > 1.5 and SIRI $\leq 1.69$; medium-risk group: $\mathrm{ALC}>1.5$ or $\mathrm{SIRI} \leq 1.69$; high-risk group: $\mathrm{ALC} \leq 1.5$ and $\mathrm{SIRI}>1.69$. Furthermore, our study also shows that post-treatment NLR $>3$ were likely to develop IRP of patients who receiving ICls. A prospective study to validate the significance of peripheral blood biomarkers is necessitated.

\section{Declarations}

\section{Ethics Approval and consent to participate}

This retrospective study was approved by the ethics committee of the Fujian Province Cancer Hospital (YK2021-009-01). All patients provided written informed consent prior to treatment, and all information was anonymized prior to analysis.

\section{Guideline statement}

All methods were carried out in accordance with relevant guidelines and regulations.

\section{Consent for publication}

Not applicable.

\section{Availability of data and materials}

The data that support the findings of this study are available from the corresponding author upon reasonable request.

\section{Competing interests}

The authors declare the submitted work was not carried out in the presence of any personal, professional or financial relationships that could potentially be construed as a conflict of interest.

\section{Contributions:}

(I) Conception and design: All authors; (II) Administrative support: None; (III) Provision of study materials or patients: All authors; (IV) Collection and assembly of data: All authors; (V) Data analysis and interpretation: All authors; (VI) Manuscript writing: All authors; (VII) Final approval of manuscript: All authors.

\section{Acknowledgments}

Not applicable. 


\section{Funding}

This study was supported in part by grants from Wu Jieping Foundation (Grant number:

320.6750.19053); Innovation of Science and Technology, Fujian Province (Grant number:2018Y9111); and the Financial Foundation of Fujian Province (Grant number: (2019)827).

\section{References}

1. Siegel RL, Miller KD, Jemal A: Cancer statistics, 2018. CA Cancer J Clin 2018, 68(1):7-30.

2. Walters S, Maringe C, Coleman MP, Peake MD, Butler J, Young N, Bergstrom S, Hanna L, Jakobsen E, Kolbeck $\mathrm{K}$ et al: Lung cancer survival and stage at diagnosis in Australia, Canada, Denmark, Norway, Sweden and the UK: a population-based study, 2004-2007. Thorax 2013, 68(6):551-564.

3. Topalian SL, Drake CG, Pardoll DM: Immune checkpoint blockade: a common denominator approach to cancer therapy. Cancer Cell 2015, 27(4):450-461.

4. Rittmeyer A, Barlesi F, Waterkamp D, Park K, Ciardiello F, von Pawel J, Gadgeel SM, Hida T, Kowalski $\mathrm{DM}$, Dols MC et al: Atezolizumab versus docetaxel in patients with previously treated non-small-cell lung cancer (OAK): a phase 3, open-label, multicentre randomised controlled trial. The Lancet 2017, 389(10066):255-265.

5. Reck M, Rodríguez-Abreu D, Robinson AG, Hui R, Csőszi T, Fülöp A, Gottfried M, Peled N, Tafreshi A, Cuffe $S$ et al: Pembrolizumab versus Chemotherapy for PD-L1-Positive Non-Small-Cell Lung Cancer. New England Journal of Medicine 2016, 375(19):1823-1833.

6. Herbst RS, Baas P, Kim D-W, Felip E, Pérez-Gracia JL, Han J-Y, Molina J, Kim J-H, Arvis CD, Ahn M-J et al: Pembrolizumab versus docetaxel for previously treated, PD-L1-positive, advanced non-small-cell lung cancer (KEYNOTE-010): a randomised controlled trial. The Lancet 2016, 387(10027):15401550.

7. Gettinger SN, Horn L, Gandhi L, Spigel DR, Antonia SJ, Rizvi NA, Powderly JD, Heist RS, Carvajal RD, Jackman DM et al: Overall Survival and Long-Term Safety of Nivolumab (Anti-Programmed Death 1 Antibody, BMS-936558, ONO-4538) in Patients With Previously Treated Advanced Non-Small-Cell Lung Cancer. J Clin Oncol 2015, 33(18):2004-2012.

8. Brahmer J, Reckamp KL, Baas P, Crino L, Eberhardt WE, Poddubskaya E, Antonia S, Pluzanski A, Vokes EE, Holgado E et al: Nivolumab versus Docetaxel in Advanced Squamous-Cell Non-Small-Cell Lung Cancer. N Engl J Med 2015, 373(2):123-135.

9. Sui H, Ma N, Wang Y, Li H, Liu X, Su Y, Yang J: Anti-PD-1/PD-L1 Therapy for Non-Small-Cell Lung Cancer: Toward Personalized Medicine and Combination Strategies. J Immunol Res 2018, 2018:6984948.

10. Michot JM, Bigenwald C, Champiat S, Collins M, Carbonnel F, Postel-Vinay S, Berdelou A, Varga A, Bahleda R, Hollebecque A et al: Immune-related adverse events with immune checkpoint blockade: a comprehensive review. Eur J Cancer 2016, 54:139-148. 
11. Nishino M, Ramaiya NH, Awad MM, Sholl LM, Maattala JA, Taibi M, Hatabu H, Ott PA, Armand PF, Hodi FS: PD-1 Inhibitor-Related Pneumonitis in Advanced Cancer Patients: Radiographic Patterns and Clinical Course. Clin Cancer Res 2016, 22(24):6051-6060.

12. Nishino M, Giobbie-Hurder A, Hatabu H, Ramaiya NH, Hodi FS: Incidence of Programmed Cell Death 1 Inhibitor-Related Pneumonitis in Patients With Advanced Cancer. A Systematic Review and Metaanalysis. JAMA Oncol 2016, 2(12):1607-1616.

13. Naidoo J, Wang X, Woo KM, lyriboz T, Halpenny D, Cunningham J, Chaft JE, Segal NH, Callahan MK, Lesokhin AM et al: Pneumonitis in Patients Treated With Anti-Programmed Death-1/Programmed Death Ligand 1 Therapy. J Clin Oncol 2017, 35(7):709-717.

14. Fukihara J, Sakamoto K, Koyama J, Ito T, Iwano S, Morise M, Ogawa M, Kondoh Y, Kimura T, Hashimoto $\mathrm{N}$ et al: Prognostic Impact and Risk Factors of Immune-Related Pneumonitis in Patients With Non-Small-Cell Lung Cancer Who Received Programmed Death 1 Inhibitors. Clin Lung Cancer 2019, 20(6):442-450 e444.

15. Grivennikov SI, Greten FR, Karin M: Immunity, inflammation, and cancer. Cel/2010, 140(6):883-899.

16. Templeton AJ, McNamara MG, Seruga B, Vera-Badillo FE, Aneja P, Ocana A, Leibowitz-Amit R, Sonpavde G, Knox JJ, Tran B et al: Prognostic role of neutrophil-to-lymphocyte ratio in solid tumors: a systematic review and meta-analysis. J Natl Cancer Inst 2014, 106(6):dju124.

17. Templeton AJ, Ace O, McNamara MG, Al-Mubarak M, Vera-Badillo FE, Hermanns T, Seruga B, Ocana A, Tannock IF, Amir E: Prognostic role of platelet to lymphocyte ratio in solid tumors: a systematic review and meta-analysis. Cancer Epidemiol Biomarkers Prev 2014, 23(7):1204-1212.

18. Shoji F, Takeoka H, Kozuma Y, Toyokawa G, Yamazaki K, Ichiki M, Takeo S: Pretreatment prognostic nutritional index as a novel biomarker in non-small cell lung cancer patients treated with immune checkpoint inhibitors. Lung Cancer 2019, 136:45-51.

19. Ksienski D, Wai ES, Alex D, Croteau NS, Freeman AT, Chan A, Patterson T, Clarkson M, Fiorino L, Poonja $Z$ et al: Prognostic significance of the neutrophil-to-lymphocyte ratio and platelet-tolymphocyte ratio for advanced non-small cell lung cancer patients with high PD-L1 tumor expression receiving pembrolizumab. Trans/ Lung Cancer Res 2021, 10(1):355-367.

20. Hu M, Xu Q, Yang S, Han S, Zhu Y, Lin Q, Sun X, Liu H, Xu Y: Pretreatment systemic inflammation response index (SIRI) is an independent predictor of survival in unresectable stage III non-small cell lung cancer treated with chemoradiotherapy: a two-center retrospective study. Ann Trans/ Med 2020, 8(20):1310.

21. Pavan A, Calvetti L, Dal Maso A, Attili I, Del Bianco P, Pasello G, Guarneri V, Aprile G, Conte P, Bonanno L: Peripheral Blood Markers Identify Risk of Immune-Related Toxicity in Advanced Non-Small Cell Lung Cancer Treated with Immune-Checkpoint Inhibitors. Oncologist 2019, 24(8):1128-1136.

22. Detterbeck FC, Boffa DJ, Kim AW, Tanoue LT: The Eighth Edition Lung Cancer Stage Classification. Chest 2017, 151(1):193-203.

23. Mantovani A, Allavena P, Sica A, Balkwill F: Cancer-related inflammation. Nature 2008, 454(7203):436-444. 
24. Lee YJ, Kim WR, Han J, Han YD, Cho MS, Hur H, Lee KY, Kim NK, Min BS: Prognostic Impact of Immunonutritional Status Changes During Preoperative Chemoradiation in Patients With Rectal Cancer. Ann Coloproctol 2016, 32(6):208-214.

25. Coussens LM, Werb Z: Inflammation and cancer. Nature 2002, 420(6917):860-867.

26. Coffelt SB, Wellenstein MD, de Visser KE: Neutrophils in cancer: neutral no more. Nat Rev Cancer 2016, 16(7):431-446.

27. Wu ES, Oduyebo T, Cobb LP, Cholakian D, Kong X, Fader AN, Levinson KL, Tanner EJ, 3rd, Stone RL, Piotrowski A et al: Lymphopenia and its association with survival in patients with locally advanced cervical cancer. Gynecol Oncol 2016, 140(1):76-82.

28. Suzuki R, Lin SH, Wei X, Allen PK, Welsh JW, Byers LA, Komaki R: Prognostic significance of pretreatment total lymphocyte count and neutrophil-to-lymphocyte ratio in extensive-stage small-cell lung cancer. Radiother Oncol 2018, 126(3):499-505.

29. Ray-Coquard I, Cropet C, Van Glabbeke M, Sebban C, Le Cesne A, Judson I, Tredan O, Verweij J, Biron P, Labidi I et al: Lymphopenia as a prognostic factor for overall survival in advanced carcinomas, sarcomas, and lymphomas. Cancer Res 2009, 69(13):5383-5391.

30. Borst J, Ahrends T, Babala N, Melief CJM, Kastenmuller W: CD4(+) T cell help in cancer immunology and immunotherapy. Nat Rev Immunol 2018, 18(10):635-647.

31. Qi Q, Zhuang L, Shen Y, Geng Y, Yu S, Chen H, Liu L, Meng Z, Wang P, Chen Z: A novel systemic inflammation response index (SIRI) for predicting the survival of patients with pancreatic cancer after chemotherapy. Cancer 2016, 122(14):2158-2167.

32. Li S, Lan X, Gao H, Li Z, Chen L, Wang W, Song S, Wang Y, Li C, Zhang H et al: Systemic Inflammation Response Index (SIRI), cancer stem cells and survival of localised gastric adenocarcinoma after curative resection. J Cancer Res Clin Oncol 2017, 143(12):2455-2468.

33. Geng Y, Zhu D, Wu C, Wu J, Wang Q, Li R, Jiang J, Wu C: A novel systemic inflammation response index (SIRI) for predicting postoperative survival of patients with esophageal squamous cell carcinoma. Int Immunopharmacol 2018, 65:503-510.

34. Chen Z, Wang K, Lu H, Xue D, Fan M, Zhuang Q, Yin S, He X, Xu R: Systemic inflammation response index predicts prognosis in patients with clear cell renal cell carcinoma: a propensity score-matched analysis. Cancer Manag Res 2019, 11:909-919.

35. Sato K, Akamatsu H, Murakami E, Sasaki S, Kanai K, Hayata A, Tokudome N, Akamatsu K, Koh Y, Ueda $\mathrm{H}$ et al: Correlation between immune-related adverse events and efficacy in non-small cell lung cancer treated with nivolumab. Lung Cancer 2018, 115:71-74.

36. Sakamoto K, Fukihara J, Morise M, Hashimoto N: Clinical burden of immune checkpoint inhibitorinduced pneumonitis. Respir Investig 2020, 58(5):305-319.

37. Nakanishi Y, Masuda T, Yamaguchi K, Sakamoto S, Horimasu Y, Nakashima T, Miyamoto S, Tsutani $\mathrm{Y}$, Iwamoto H, Fujitaka $\mathrm{K}$ et al: Pre-existing interstitial lung abnormalities are risk factors for immune checkpoint inhibitor-induced interstitial lung disease in non-small cell lung cancer. Respir Investig 2019, 57(5):451-459. 
38. Suresh K, Voong KR, Shankar B, Forde PM, Ettinger DS, Marrone KA, Kelly RJ, Hann CL, Levy B, Feliciano JL et al: Pneumonitis in Non-Small Cell Lung Cancer Patients Receiving Immune Checkpoint Immunotherapy: Incidence and Risk Factors. J Thorac Oncol 2018, 13(12):1930-1939.

39. Cui P, Liu Z, Wang G, Ma J, Qian Y, Zhang F, Han C, Long Y, Li Y, Zheng X et al: Risk factors for pneumonitis in patients treated with anti-programmed death-1 therapy: A case-control study. Cancer Med 2018, 7(8):4115-4120.

40. Matsukane R, Watanabe H, Minami H, Hata K, Suetsugu K, Tsuji T, Masuda S, Okamoto I, Nakagawa $\mathrm{T}$, Ito $\mathrm{T}$ et al: Continuous monitoring of neutrophils to lymphocytes ratio for estimating the onset, severity, and subsequent prognosis of immune related adverse events. Sci Rep 2021, 11(1):1324.

41. Fujisawa Y, Yoshino K, Otsuka A, Funakoshi T, Fujimura T, Yamamoto Y, Hata H, Gosho M, Tanaka R, Yamaguchi $\mathrm{K}$ et al: Fluctuations in routine blood count might signal severe immune-related adverse events in melanoma patients treated with nivolumab. J Dermato/ Sci 2017, 88(2):225-231.

\section{Figures}

A

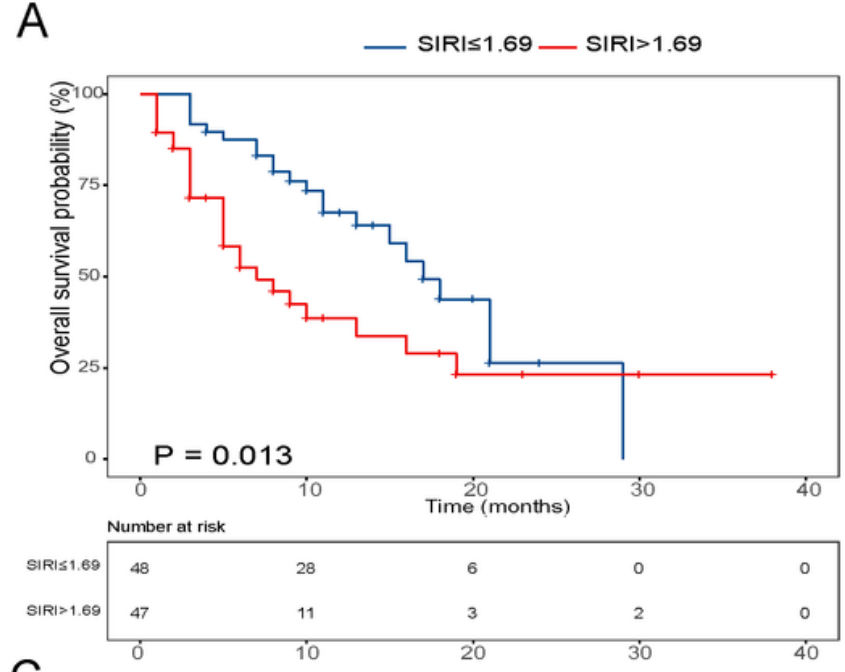

C

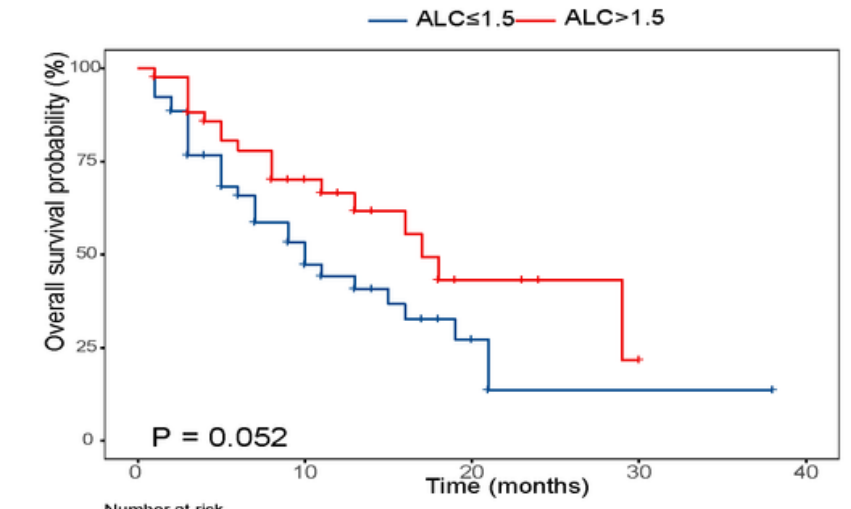

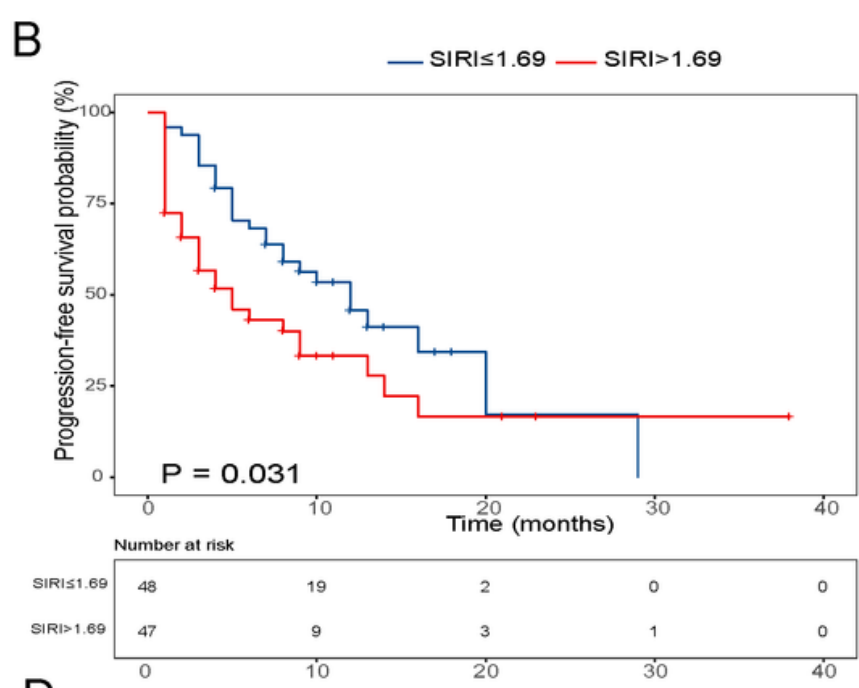

D
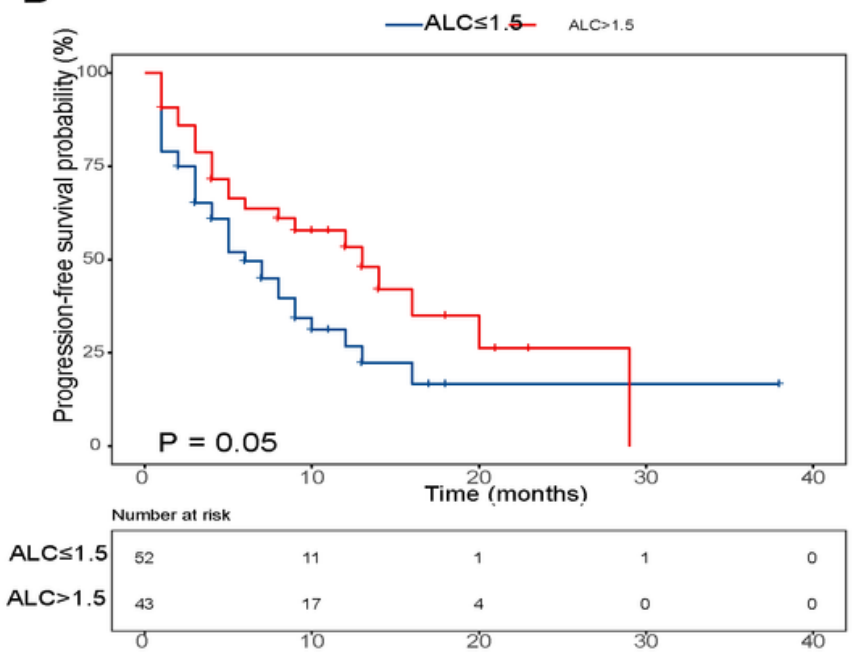

\section{Figure 1}


Analysis of overall survival for patients according to post-treatment systemic inflammation response index (SIRI) (A), pretreatment absolute lymphocyte count (ALC) (C), and analysis of progression-free survival for patients according to post-treatment SIRI (B), pretreatment ALC (D).

A

— low-risk — medium-risk — high-risk

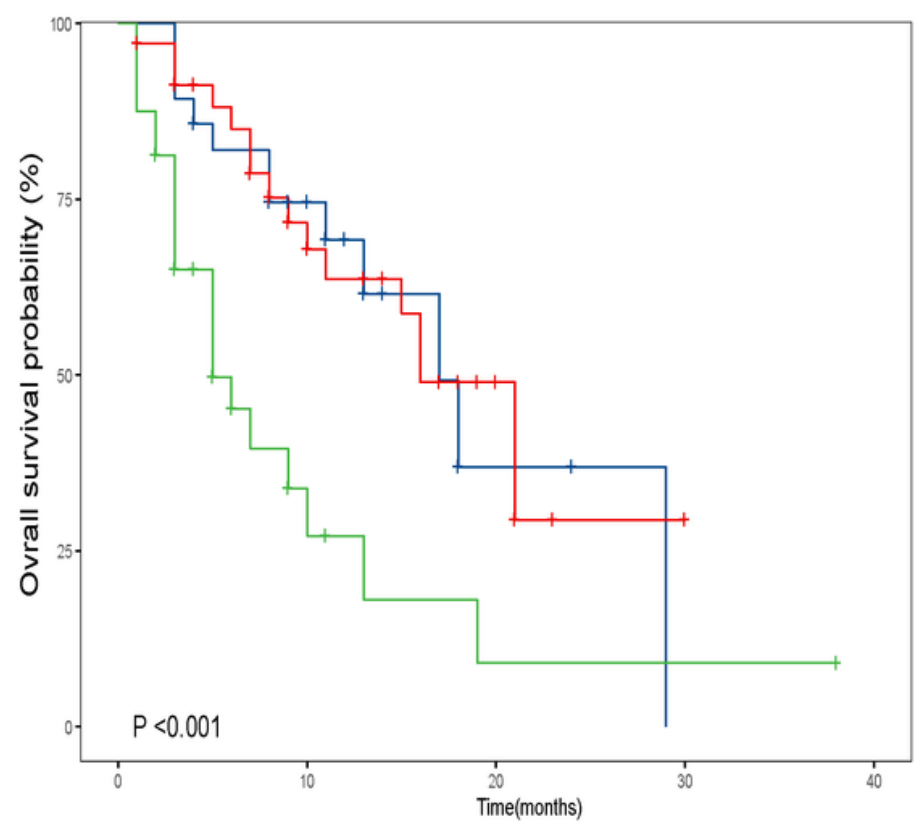

Number at risk

\begin{tabular}{l|ccccc|}
\cline { 2 - 6 } low & 28 & 15 & 2 & 0 & 0 \\
medium & 35 & 19 & 6 & 1 & 0 \\
high & 32 & 5 & 1 & 1 & 0 \\
\hline
\end{tabular}

B
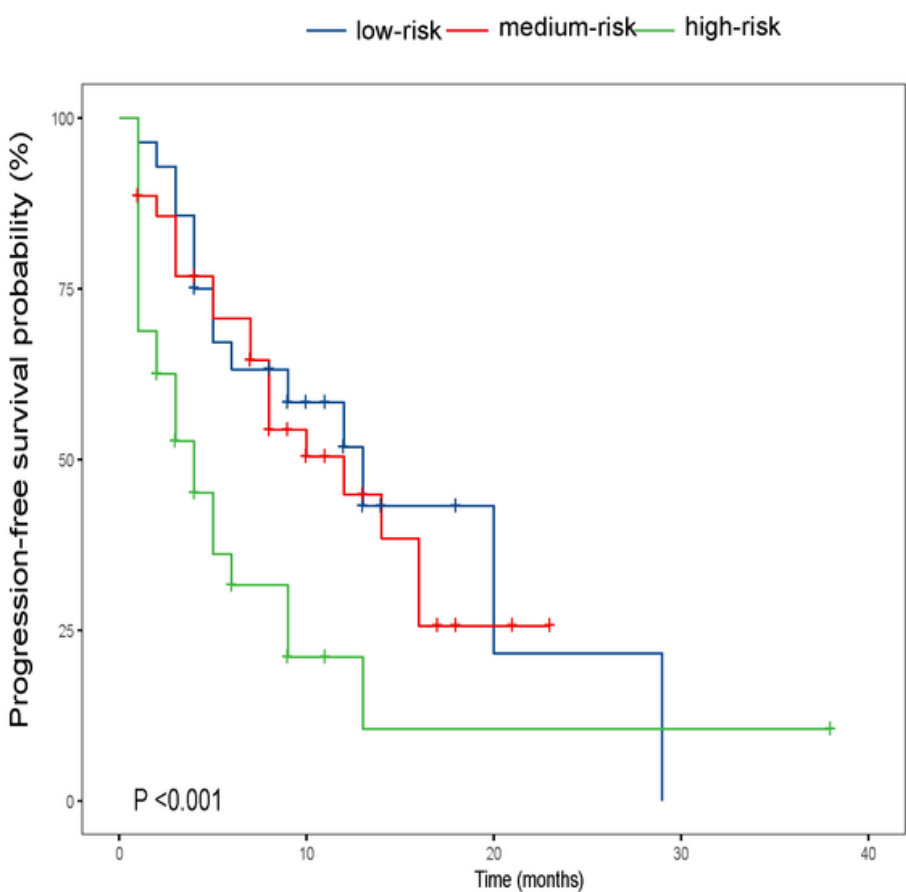

Number at risk

\begin{tabular}{l|ccc|c|c|}
\cline { 2 - 5 } low & 28 & 11 & 2 & 0 & 0 \\
high & 14 & 2 & 0 & 0 \\
\hline 35 & 3 & 1 & 1 & 0 \\
\hline & 1 & 1 & 1 & 30 & 40
\end{tabular}

\section{Figure 2}

Overall survival (A) and Progression-free survival (B) curves of the risk stratification according to posttreatment systemic inflammation response index (SIRI) and pretreatment absolute lymphocyte count.

A

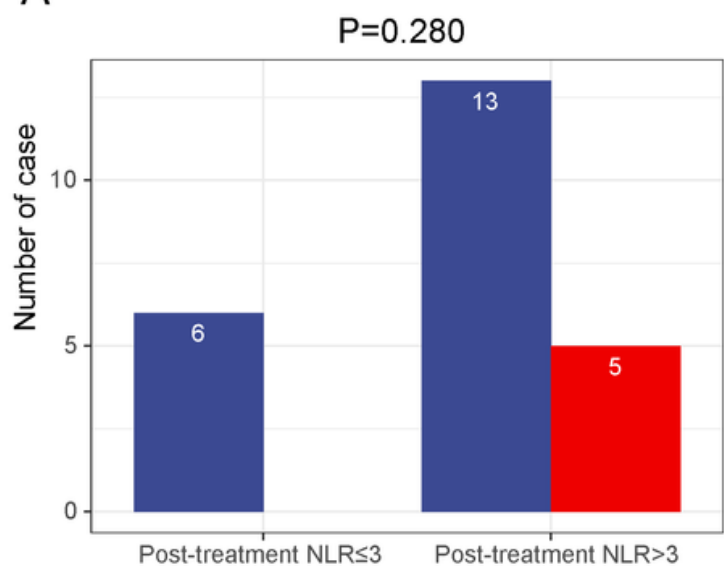

B

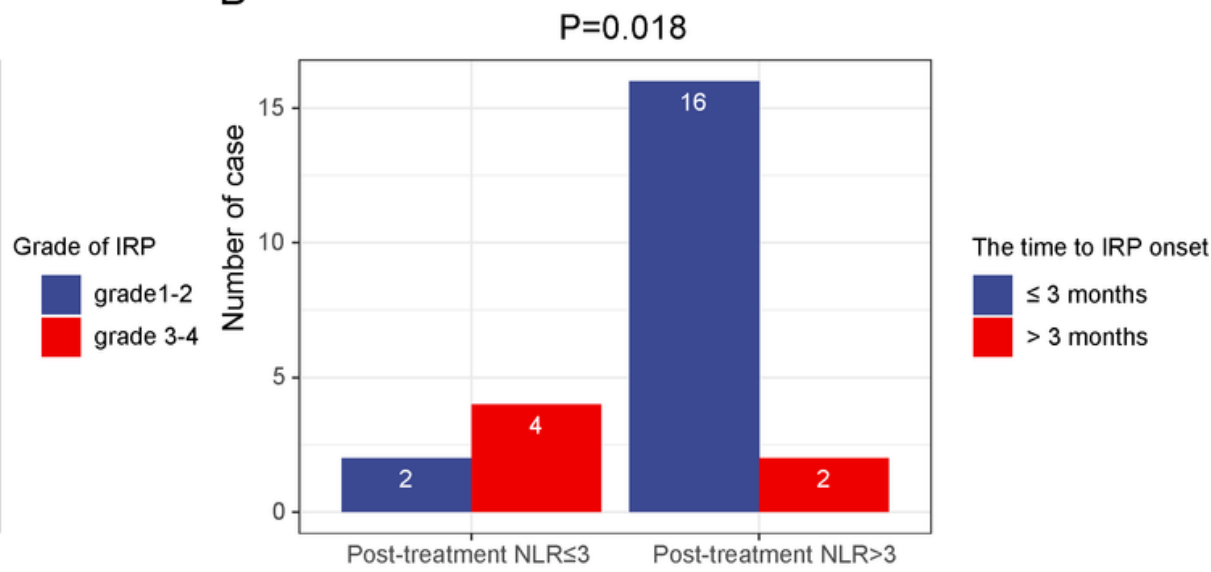


Figure 3

Comparison of the grade distribution of immune-related pneumonitis (IRP) in the patients with posttreatment neutrophil-to-lymphocyte ratio $(\mathrm{NLR}) \leq 3$ and $>3(\mathrm{~A})$, and comparison of the time to develop IRP in the patients with post-treatment NLR $\leq 3$ and $>3$.

A
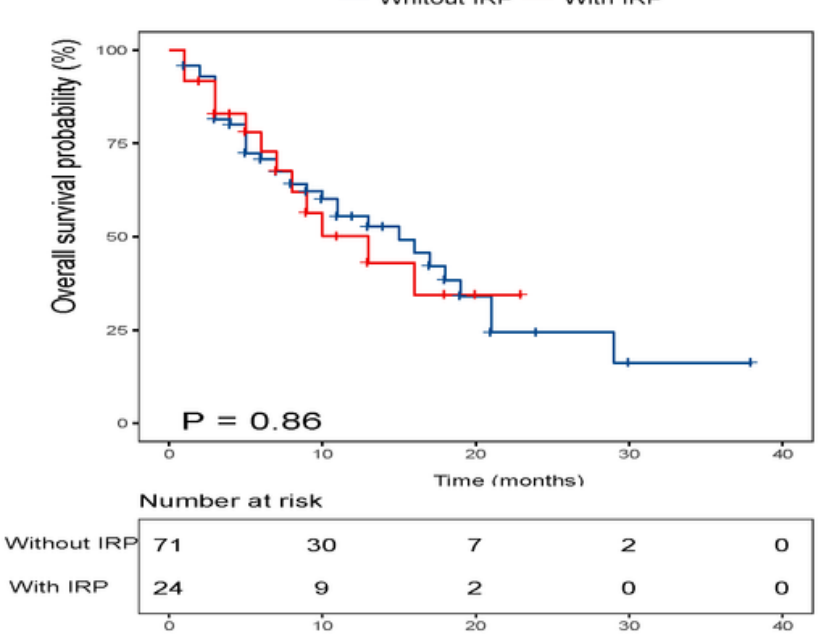

C

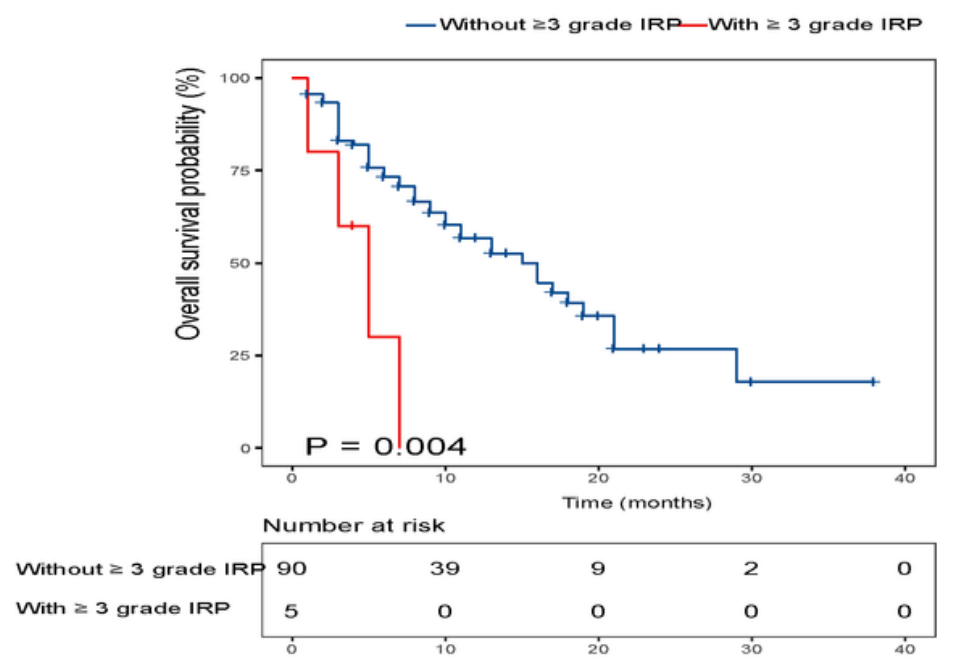

B
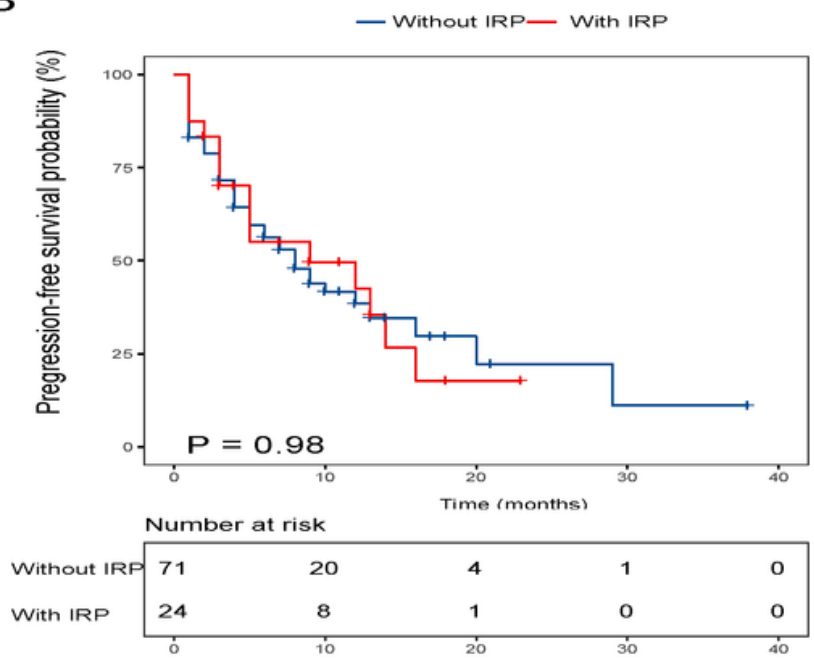

D

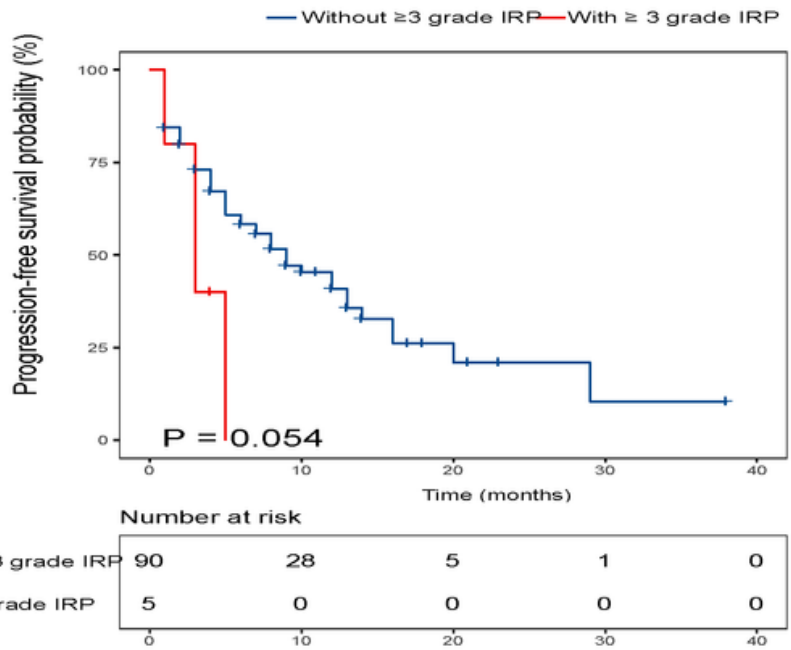

\section{Figure 4}

Analysis of overall survival (OS) (A) and progression-free survival (PFS) (B) for patients with or without immune-related pneumonitis (IRP), and analysis of OS (C) and PFS (D) for patients according to the grades of IRP. 